\title{
Editorial
}

\section{Nursing research: a key to quality care}

People who need nursing deserve good quality care. To determine what are the most appropriate interventions sound research is required. The findings of such research studies increase the sum of our knowledge and skills so that the service to clients, their families, and the public in general can continually improve. However, in terms of research based practice, nursing is still in its infancy. Until recently, nurses were not active in research nor educated in a culture where research was seen as necessary. Perhaps many others also do not have the expectation that nursing interventions should be tested. Indeed, this may be one of the factors that is limiting nursing research. Although medical research has a much longer history, medical interventions are not always based on good research. Proctor stated that "clinical research is considered by many doctors as erudite and not an activity likely to involve them directly ... most practitioners are so distanced from clinical research."

The main focus of nursing research must be to develop and test best practice. In many instances the studies are concerned with the patient and his or her family's responses to actual or potential illness rather than the illness itself. When the vulnerability of people facing or experiencing illness is considered the results of such research can have a profound effect on the quality of care. These issues and much of the other patient centred work that nurses do cannot easily be expressed in quantifiable research terms.

It is almost a given that prevention and treatment of pressure sores come within the domain of nursing. Most nurses and others would consider that a plethora of good nursing studies in this topic exist and that they form the basis for subsequent care. But a comprehensive review by Cullum et al in the Effectiveness Bulletin in this issue ( $\mathrm{p} 289$ ) highlights the insufficiency and inadequacy of studies of how preventive appliances are used and how interventions such as risk assessment and repositioning affect pressure area breakdown. ${ }^{2}$ The review's findings are disturbing and offer a challenge to nurse researchers generally. Much nursing research remains to be carried out, previous studies need to be replicated, and the non-cumulative nature of many investigations has to be addressed. We need to build a programme of research of nursing care, showing that nurses and nursing research can make a substantial contribution to the health of the nation.

\section{Researching effectiveness and efficiency of nursing care}

Those groups of professionals who can show evidence of the effectiveness and efficiency of the service they provide will be in a better position to survive in the National Health Service marketplace. Nurses, who account for the largest slice of health service expenditure, are being disadvantaged when it comes to accumulating such evidence. They are underrepresented on grant awarding bodies and, as a result, are underfunded and underactive in researching clinical outcomes achieved for resources allocated. In 1991 Butterworth noted that not only was funding difficult to obtain there were also no easily identifiable sources for financing nursing research. ${ }^{3}$ The situation has not improved greatly in the intervening years. In fact, it is possible that in a more competitive health service, nursing research monies may become even more difficult to obtain.

The government, and commissioning bodies generally, seem to view nursing research and nurse researchers as undeserving of major research funding. Finance may be available to test a new drug or a new piece of technology, but where does one look for money to evaluate the effects of touch on terminally ill people, or play on an autistic child, or the use of compassion or empathy on a person with schizophrenia? These, and many other similar caring interventions are making a major difference to the quality of peoples' welfare but they, and the qualitative research methods favoured by many nurse researchers, have low priority within research and development budgets.

Further, nurses cannot afford to compartmentalise themselves totally as unidisciplinary researchers, nor to merely act as data collectors for doctors. The research and development strategy calls for more multidisciplinary research. ${ }^{4}$ Although this could be a way for nurses to gain access to scarce research funds, it could also mean that research of those phenomena of specific interest to nursing will be sacrificed for the sake of multidisciplinary research collaboration. Therefore, to get experience in research and to acquire substantial grants nurses may have to study non-nursing topics - not something other health professionals would savour.

\section{Expensive option}

In the United States the government has recognised the importance of supporting nursing research through the award of substantial grants. In Europe this has not occurred to any great extent. This is short sighted considering that poor care is expensive and research activities can lead to saving money on outmoded practices and to improvements in quality of care. Governments and commissioning bodies should heed the following paraphrase of Bok: "if you think research is expensive try ignorance!". 5

Nurses are currently involved in many quality improvement initiatives, including setting standards for best practice. These standards and the practice emanating from them should be based on sound clinical research findings. If such research is lacking or insubstantial the basis for audit will be routines and rituals - hardly a sound foundation for quality improvement generally. There must therefore be an imperative to develop a coherent programme of robust nursing research valued by all.

HUGH McKENNA Senior Lecturer, Nursing

EAMON SLEVIN Lecturer, Nursing

School of Health Sciences,

University of Ulster,

fordanstown, Country Antrim,

Northern Ireland BT37 OQB

1 Proctor SJ. Why clinical research needs medical audit. Quality in Health Care 1993;3(2):1-2.

2 Cullum N, Deeks JJ, Fletcher AW, Sheldon TA, Song F. Preventing and treating pressure sores. Quality in Health Care 1995;4:289-297.

Butterworth T. Generating research in mental health nursing. International fournal of Studies 1991;28:237-46.

Fournal of Studies 1991;28:237-46.

5 Bok. In: Oxford dictionary of quotations. 3rd ed. London: Guild Publishing, 1991. 